\title{
Quantum Entaglement Entropy Produces Energy By Info-Entropy Fields Forces
}

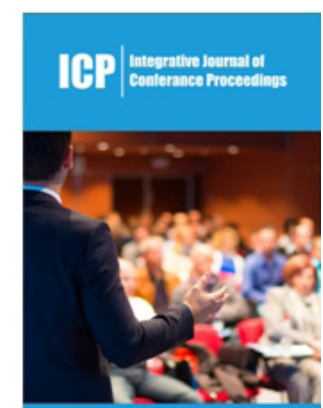

*Corresponding author: Robert Skopec, Researcher-analyst, Dubnik, Slovakias

Submission: 海 August 19, 2019

Published: 洞August 22, 2019

Volume 2 - Issue 1

How to cite this article: Robert Skopec Quantum Entaglement Entropy Produces Energy By Info-Entropy Fields Forces. Int J Conf Proc.2(1). ICP.000524.2019.

Copyright@ Robert Skopec, This article is distributed under the terms of the Creative Commons Attribution 4.0 International License, which permits unrestricted use and redistribution provided that the original author and source are credited.
Robert Skopec*

Researcher-analyst, Slovakia

\begin{abstract}
Sometimes, if you want to understand how nature truly works, you need to break things down to the simplest levels imaginable. The macroscopic world is composed of particles that are-if you divide them until they can be divided no more-fundamental. They experience forces that are determined by the exchange of additional particles (or the curvature of spacetime, for gravity), and react to the presence of objects around them. At least, that's how it seems. The closer two objects are, the greater the forces they exert on one another. If they're too far away, the forces drop off to zero, just like your intuition tells you they should. This is called the principle of locality, and it holds true in almost every instance. But in quantum mechanics, it's violated all the time. Locality may be nothing but a persistent illusion, and seeing through that facade may be just what physics needs.
\end{abstract}

Keywords: Quantum gravity, Principle of non-locality, Einstein's general theory of relativity, Quantum entanglement entropy (QEE); Schrödinger's cat; Superposition; Information about its entangled partner; Teleport information; Quantum physics as fundamentally a non-local theory

\section{Introduction}

Everybody knows holograms from credit cards or banknotes. They are two dimensional, but to us they appear three dimensional. Our Universe could behave quite similarly. In 1997, the physicist Juan Maldacena proposed the idea that there is a correspondence between gravitational theories in curved anti-de-sitter spaces on the one hand and quantum field theories in spaces with one fewer dimension on the other [1]. If quantum gravity in a flat space allows for holographic description by standard quantum theory, then there must be physical quantities, which can be calculated in both theories-and the results must agree. Especially one key feature of quantum mechanics-quantum entanglement-has to appear in the gravitational theory [2].

When quantum particles are entangled, they cannot be described individually. They form a single quantum object, even if they are located far apart. There is a measure for the amount of entanglement in quantum system, called „entropy of entanglement“. This entropy of entanglement takes the same value in flat quantum gravity and in a low dimension quantum field theory. This calculation affirms our assumption that the holographic principle can also be realized in flat spaces. I tis evidence for the validity of this correspondence in our Universe. The fact that we can even talk about quantum information and entropy of entanglement in a theory of gravity is astounding in itself, and would hardly have been imaginable only a few years back. Apparently there is growing evidence for the validity of the correspondence principle inour own Universe $[3,4]$.

\section{Spacetime is built from quantum entanglement}

Now are published papers about a significant step toward unifying general relativity and quantum mechanics by explaining how spacetime emerges from quantum entanglement. However, understanding the precise mechanics for the emergence of three-dimensional volume from the two-dimensional surface has been not easy. Hirosi Ooguri, from the University of Tokyo, and Caltech mathematician Matilde Marcolli, argue that quantum entanglement is the key to solve this question $[5,6]$. Using a quantum theory(that does not include gravity), they showed how to compute energy density, which is a source of gravitational interactions in three dimensions, using quantum entanglement data on the surface. This is analogous to diagnosing conditions inside of your body by looking at X-ray images on two-dimensional sheets. This allowed to interpret universal properties of quantum entanglement as conditions 
on the energy density that should be satisied by any consistent quantum theory of gravity, without actually explicitly including gravity in the theory of Ooguri and Marcolli: that is quantum entanglement generates the extra dimensions of the gravitational theory $[7,8]$.

It was known that quantum entanglement is related to deep issues in unification of general relativity and quantum mechanics, such as the black hole information paradox and the firewall paradox. This sheds new light on the relation between quantum entanglement and the microscopic structure of spacetime by explicit calculations. The interface between quantum gravity and information science is becoming increasingly important for both fields. I myself am collaborating with information scientists to pursue this line of research further $[8,9]$.

\section{Karma and quantum mechanics}

Whether you're into Eastern philosophy or Western scientific fact-finding, both disciplines strive to tackle the same goal: understanding how the universe works. For centuries, Buddhists have tackled this age-old question of the inner workings of the universe and have a wonderfully simplified approach to it-they call it the law of Karma [10]. Westerners, on the other hand, have created a relatively new field of scientific study called quantum mechanics, seeking to derive mathematical formulas to capture the universe at work.

Here's the thing, they both agree on a fundamental: everything we do creates a corresponding energy that comes back to us in some form or another. Think of it this way: I hold a door for someone, and that someone passes along that action to someone else by holding the door for them [11].

\section{How do i get what i want?}

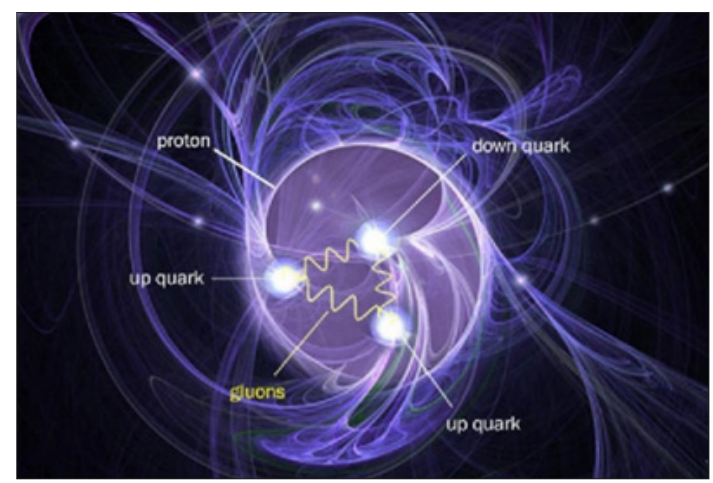

Figure 1.

I'll be the first to admit that I am no scientist, nor am I a Buddhist monk, but I'm fascinated by both disciplines and have spent some time studying them. If you embrace the fact that, from a scientific perspective, the energy you expend taking an action comes back to you or someone else, then you'll get where I'm going with this article. Ask any Buddhist monk this question, "How do I get what I want?" They will respond, (very calmly and matter-of-factly) "Help someone else get the same thing." The proton's structure, modeled along with its attendant fields, show how even though it's made out of point-like quarks and gluons, it has a finite, substan- tial size which arises from the interplay of the quantum forces and fields inside it. The proton, itself, is a composite, not fundamental, quantum particle. (Brookhaven national laboratory) A single thought experiment reveals a paradox. As part of Quantum Gravity theory, the Quantum Entanglement Entropy (QEE) is the solution (Figure 1).

\section{The bigger the challenge, the bigger the effort is required}

The universe doesn't immediately respond to your actions with good Karma. It can take time before the universe repays your intentional actions with more actions that help you progress toward your goal. Like a slingshot, the universe requires energy to be expended, such as pulling back a slingshot. You might find yourself generating days, if not weeks or months, of output before you see the effects of your efforts. Sometimes, the efforts come in a trickle, and other times, they can come in deluge [12]. The trick is to keep your focus on generating actions that help others in the direction you seek to go yourself.

\section{Nothing takes the place of committed all-in action every day}

One more thought: this topic that I'm introducing is barely the tip of the iceberg of the meta-physical dynamics of the universe in which we live. But the ideas are relevant to anyone seeking to turn a dream into reality. There are forces at work that can help or hinder your efforts. The more you put forth energies without expectation of personal gain, while helping others, the more you'll be surprised at how the universe will open the door to the goal you seek to attain. Make no mistake, nothing takes the place of committed All-in action every day. Never, Ever, Give Up on your dreams, and soon you'll discover that the universe will not give up on you $[13,14]$. Quantum gravity tries to combine Einstein's general theory of relativity with quantum mechanics (Figure 2). Quantum corrections to classical gravity are visualized as loop diagrams, as the one shown here in white. We typically view objects that are close to one another as capable of exerting forces on one another, but that might be an illusion, too. (slac national accelerator lab) imagine that you had two objects located in close proximity to one another. They would attract or repel one another based on their charges and the distance between them. You might visualize this as one object generating a field that affects the other, or as two objects exchanging particles that impart either a push or a pull to one or both of them.

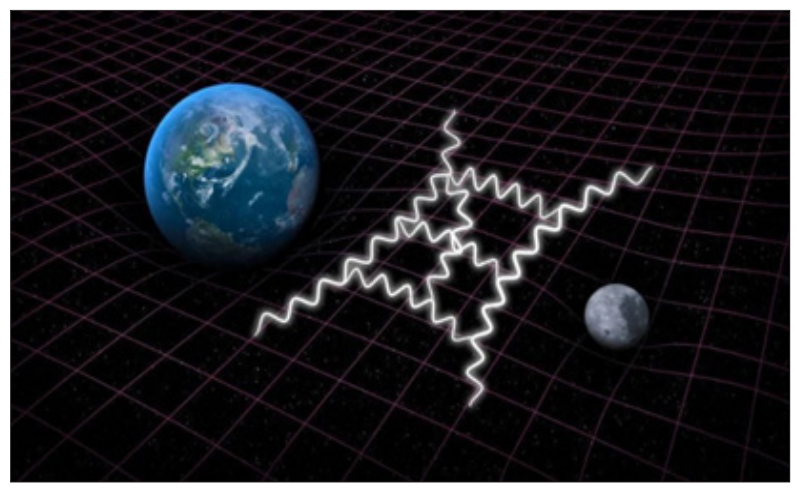

Figure 2. 
You'd expect, of course, that there would be a speed limit to this interaction: the speed of light. Relativity gives you no other way out, since the speed at which the particles responsible for forces propagate is limited by the speed they can travel, which can never exceed the speed of light for any particle in the Universe. It seems so straightforward, and yet the Universe is full of surprises (Figure 3). An example of a light cone, the three-dimensional surface of all possible light rays arriving at and departing from a point in spacetime. The more you move through space, the less you move through time, and vice versa. Only things contained within your past light-cone can affect you today; only things contained within your future light-cone can be perceived by you in the future(wikimedia commons user missmj).

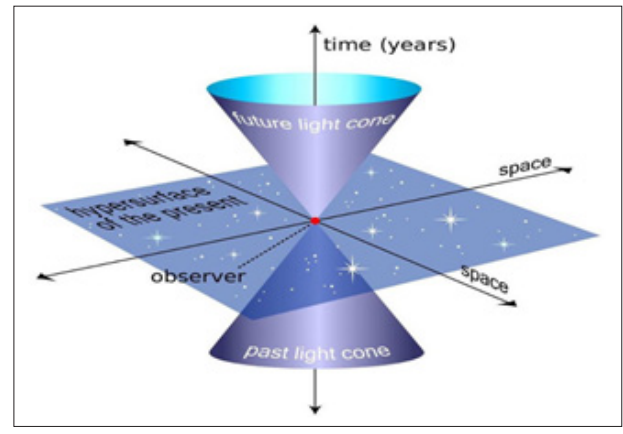

Figure 3.

We have this notion of cause-and-effect that's been hard-wired into us by our experience with reality. Physicists call this causality, and it's one of the rare physics ideas that actually conforms to our intuition. Every observer in the Universe, from its own perspective, has a set of events that exist in its past and in its future [15]. In relativity, these are events contained within either your past lightcone (for events that can causally affect you) or your future lightcone (for events that you can causally effect). Events that can be seen, perceived, or can otherwise have an effect on an observer are known as causally-connected. Signals and physical effects, both from the past and into the future, can propagate at the speed of light, but no faster. At least, that's what your intuitive notions about reality tell you (Figure 4).

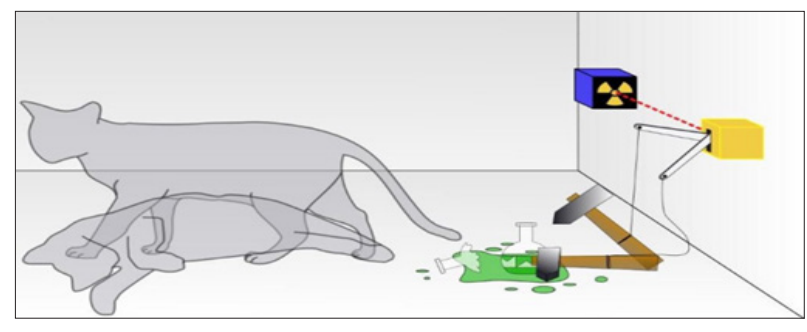

Figure 4.

Schrödinger's cat. Inside the box, the cat will be either alive or dead, depending on whether a radioactive particle decayed or not. If the cat were a true quantum system, the cat would be neither alive nor dead, but in a superposition of both states until observed. (wikimedia commons user dhatfield). but in the quantum Universe, this notion of relativistic causality isn't as straightforward or universal as it would seem. There are many properties that a particle can have-such as its spin or polarization-that are fundamentally indeterminate until you make a measurement. Prior to observing the particle, or interacting with it in such a way that it's forced to be in either one state or the other, it's actually in a superposition of all possible outcomes [16].

Well, you can also take two quantum particles and entangle them, so that these very same quantum properties are linked between the two entangled particles. Whenever you interact with one member of the entangled pair, you not only gain information about which particular state it's in, but also information about its entangled partner (Figure 4). By creating two entangled photons from a preexisting system and separating them by great distances, we can 'teleport' information about the state of one by measuring the state of the other, even from extraordinarily different locations(melissa meister, of laser photons through a beam splitter).

This wouldn't be so bad, except for the fact that you can set up an experiment as follows. You can create your pair of entangled particles at a particular location in space and time. You can transport them an arbitrarily large distance apart from one another, all while maintaining that quantum entanglement. Finally, you can make those measurements (or force those interactions) as close to simultaneously as possible. In every instance where you do this, you'll find the member you measure in a particular state, and instantly "know" some information about the other entangled member (Figure 5).

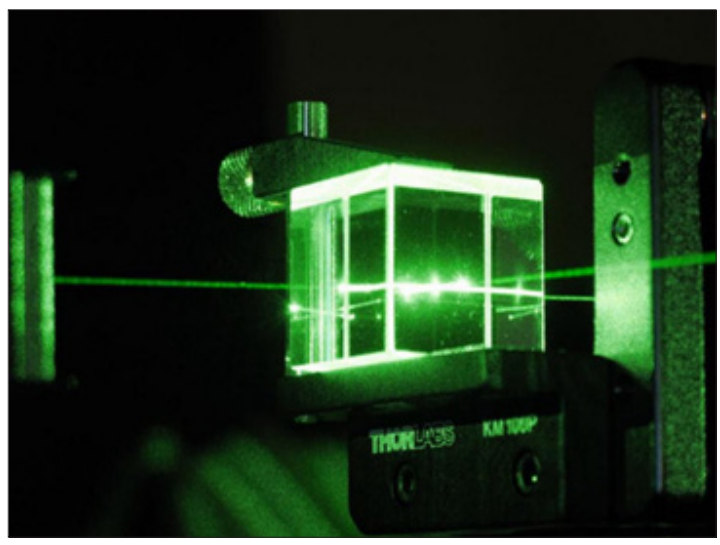

Figure 5.

A photon can have two types of circular polarizations, arbitrarily defined so that one is + and one is -. By devising an experiment to test correlations between the directional polarization of entangled particles, one can attempt to distinguish between certain formulations of quantum mechanics that lead to different experimental results(Dave3457/wikimedia commons). What's puzzling is that you cannot check whether this information is true or not until much later, because it takes a finite amount of time for a light signal to arrive from the other member. When the signal does arrive, it always confirms what you'd known just by measuring your member of the entangled pair: your expectation for the state of the distant particle agreed $100 \%$ with what its measurement indicated. Only, there seems to be a problem [17]. You "knew" information about a measurement that was taking place non-locally, which is to 
say that the measurement that occurred is outside of your light cone. Yet somehow, you weren't entirely ignorant about what was going on over there. Even though no information was transmitted faster than the speed of light, this measurement describes a troubling truth about quantum physics: It is fundamentally a non-local theory (Figure 6 \& 7). Schematic of the third Aspect experiment testing quantum non-locality. Entangled photons from the source are sent to two fast switches that direct them to polarizing detectors. The switches change settings very rapidly, effectively changing the detector settings for the experiment while the photons are in flight (chad orzel).

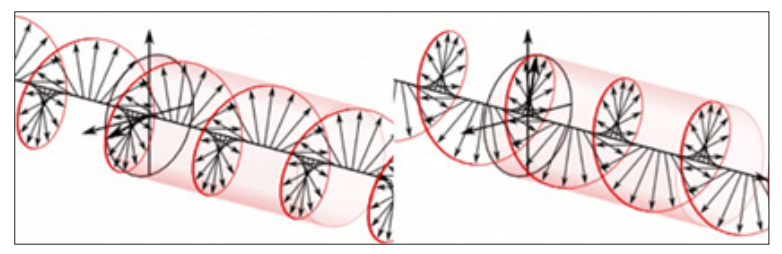

Figure 6.

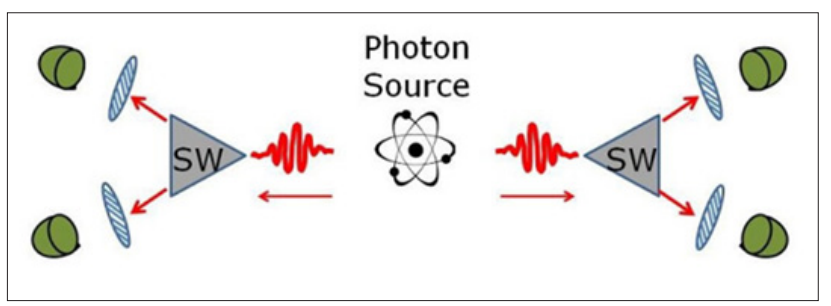

Figure 7.

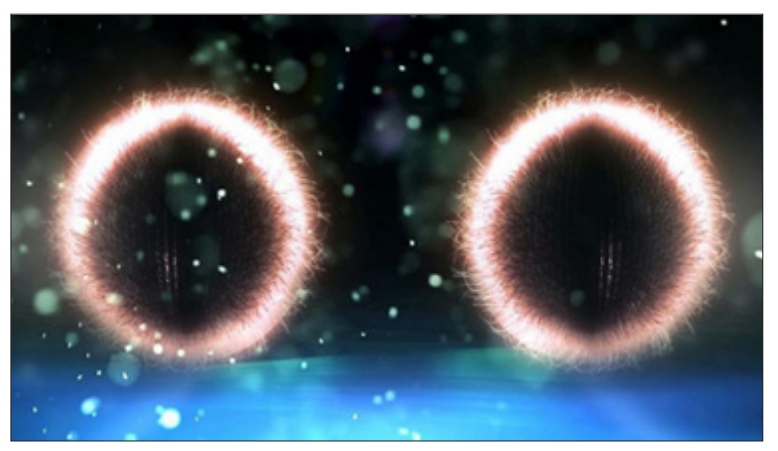

Figure 8.

There are limits to this, of course. It isn't as clean as you want: measuring the state of your particle doesn't tell us the exact state of its entangled pair, just probabilistic information about its partner. There is still no way to send a signal faster than light; you can only use this non-locality to predict a statistical average of entangled particle properties. And even though it has been the dream of many, from Einstein to Schrödinger to de Broglie, no one has ever come up with an improved version of quantum mechanics that tells you anything more than its original formulation [18]. But there are many who still dream that dream. If two particles are entangled, they have complementary wavefunction properties, and measuring one places meaningful constraints on the properties of the other (Figure 8). (wikimedia commons user david koryagin) One of them is Lee Smolin, who cowrote a paper [Physical Review D] way back in 2003 that showed an intriguing link between general ideas in quantum gravity and the fundamental non-locality of quantum physics. Although we don't have a successful quantum theory of gravity, we have established a number of important properties concerning how a quantum theory of gravity will behave and still be consistent with the known Universe (Figure 9).

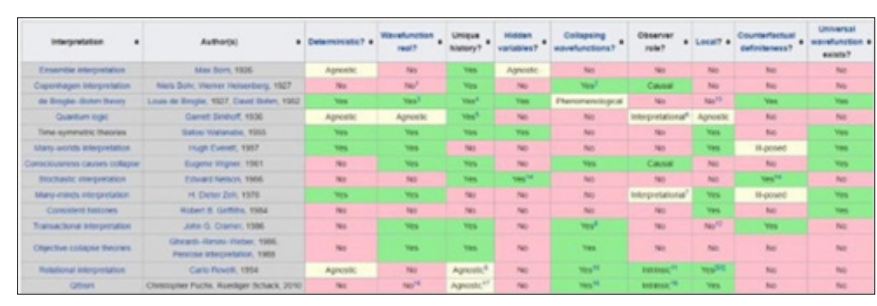

Figure 9.

A variety of quantum interpretations and their differing assignments of a variety of properties. Despite their differences, there are no experiments known that can tell these various interpretations apart from one another, although certain interpretations, like those with local, real, deterministic hidden variables, can be ruled out. (English wikipedia page on interpretations of quantum mechanics) There are many reasons to be skeptical that this conjecture will hold up to further scrutiny. For one, we don't truly understand quantum gravity at all, and anything we can say about it is extraordinarily provisional. For another, replacing the non-local behavior of quantum mechanics with the non-local behavior of quantum gravity is arguably making the problem worse, not better. And, as a third reason, there is nothing thought to be observable or testable about these non-local variables that Markopoulou and Smolin claim could explain this bizarre property of the quantum Universe [19].

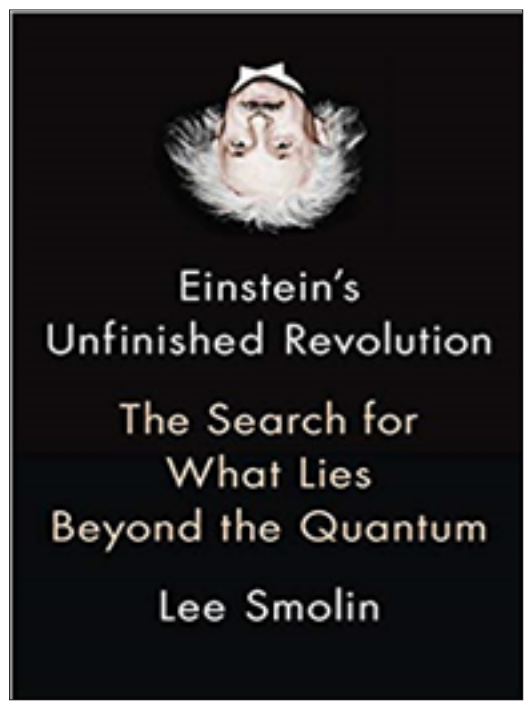

Figure 10.

Fortunately, we'll have the opportunity to hear the story direct from Smolin himself and evaluate it on our own. It is curious about what Smolin is calling Einstein's Unfinished Revolution, which is the ultimate quest to supersede our two current (but mutually incompatible) descriptions of reality: General Relativity and quantum mechanics (Figure 10). Find out where we are in the quest 
for quantum gravity, and what promises it may (or may not) have for revolutionizing one of the greatest counterintuitive mysteries about the quantum nature of reality! $[19,20]$ Thanks for joining me for an interesting lecture and discussions on science, and just maybe, someday, we'll have some interesting progress to report on this topic. Until then, you don't have to shut up, but you still do have to calculate!

\section{Quantum entanglement entropy plays a key role}

Gauge/gravity duality posits an exact equivalence between certain conformal field theories (CFT's) with many degrees of freedom and higher dimensional theories with gravity. We try to understand how bulk spacetime geometry and gravitational dynamics emerge from a non-gravitational theory. In recent years, there have appeared hints that quantum entanglement entropy a key role. One important development in this direction was the proposal that the entanglement entropy between spatial domain $\mathrm{D}$ of CFT and its complement is equal to the area of the bulk extremal surface. Using this showed the emergence of linearized gravity from entanglement physics of the CFT, we continue this program. Moreover, we show that bulk stress-energy density in this region can be reconstructed point-by-point from entanglement on the boundary [21].

Relative entropy is a measure of distinguishability between two quantum state in the Hilbert space. The relative entropy of two density matrices $p_{0}$ and $p_{1}$ is defined as

$$
S\left(p_{0} / p_{1}\right)=\operatorname{tr}\left(p_{1} \log p_{1}\right)-\operatorname{tr}\left(p_{1} \log p_{0}\right)
$$

When $p_{0}$ and $p_{1}$ are reduced density matrices on a spatial domain D for two states of a quantum field theory (QFT), which is the case which implies that $\mathrm{S}\left(p_{0} / p_{1}\right)$ increases with the size of D.

Defining the ${ }_{e^{-H} \text { mod }}^{\operatorname{modu}}$ Hamiltonial of $H_{\text {mod }} p_{0}$ implicitly through $p_{0}=p_{0}=\frac{e^{-H_{\text {mad }}}}{\operatorname{tr}\left(e^{\left.-H_{\text {mad }}\right)}\right)}$ It is easy to see that above is equivalent to $s\left(p_{0} / p_{1}\right)=\Delta\left(H_{\bmod }\right)-\Delta S_{E E} \geq 0$ where $\Delta\left(H_{\text {mod }}=\operatorname{tr}\left(p_{1} H_{\text {mod }}\right)-\operatorname{tr}\left(p_{0} H_{\text {mod }}\right)\right.$ where is the change in the expectation value of the operator $H_{\bmod }$

and $\Delta s=-\operatorname{tr}\left(p_{1} \log p_{1}\right)-\operatorname{tr}\left(p_{0} \log p_{0}\right)$ is the change in entanglement entropy across $\mathrm{D}$ as one goes between the states.

In general, the modular Hamiltonian associated to a given density matrix is nonlocal. There are a few simple cases where it is explicitly known. When $p_{0}$ is the reduced density matrix of the vacuum state of a CFT on a disk of radius $\mathrm{R}$ which (without loss of generality) we take to be centered at $x_{0}=0$

$$
H_{\text {mod }}=\pi \int d^{d-1} x \frac{R^{2}-x^{2}}{R} T_{t t}(x)
$$

where is the energy density of the CFT [22].

\section{The Interface between quantum gravity and information science}

Theory of quantum gravitation: Lee Smolin showed an intriguing link between general ideas in quantum gravity and the fundamental non-locality of quantum physics, We must replace the non-local behavior of quantum mechanics with the non-local behavior of quantum gravity. [23].

Quantum entanglement entropy: Ooguri and Marcolli's work shows that this quantum entanglement generates the extra dimensions of the gravitational theory, entangled particles have also complementary properties, entangled quantum particles cannot be seen individually, they form a single quantum object, even if they are located far apart, If two particles are entangled they have complementary wavefunction properties and measuring one places meaningful constraints on the properties of the other.

Quantum Information: The interface between quantum gravity and information science is becoming increasingly important for both fields [24]. based on lee smolin's calling for continuing in einstein's unfinished revolution, i propose the ultimate quest to supersede our two current (mutually compatible) descriptions of reality: general relativity and quantum gravity. general relativity and quantum gravity including quantum entanglement. entropy means that the twofaced new main law of nature may lead to a new scientific revolution.

\section{Conclusion}

It has been shown that information and "entropy"-a measure of the disorder of a system-are linked together to "infoentropy" in a way exactly analogous to electric and magnetic fields ("electromagnetism"). Electric currents produce magnetic fields, while changing magnetic fields produce electric currents. Information and entropy influence each other in the same way.

Entropy is a fundamental concept in physics. For example, because entropy can never decrease (disorder always increases) you can turn an egg into scrambled eggs but not the other way around. If you move information around you must also increase entropy-a phone call has an entropy cost. It has been showed that entropy and information can be treated as a field and that they are related to geometry. Think of the two strands of the DNA double helix winding around each other. Light waves have the same structure, where the two strands are the electric and magnetic fields. We showed mathematically that the relationship between information and entropy can be visualised using just the same geometry (Figure $11 \& 12$ ).

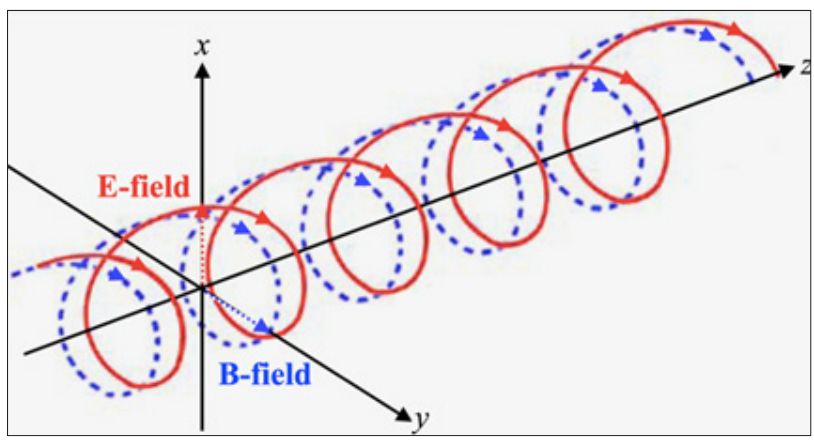

Figure 11. 


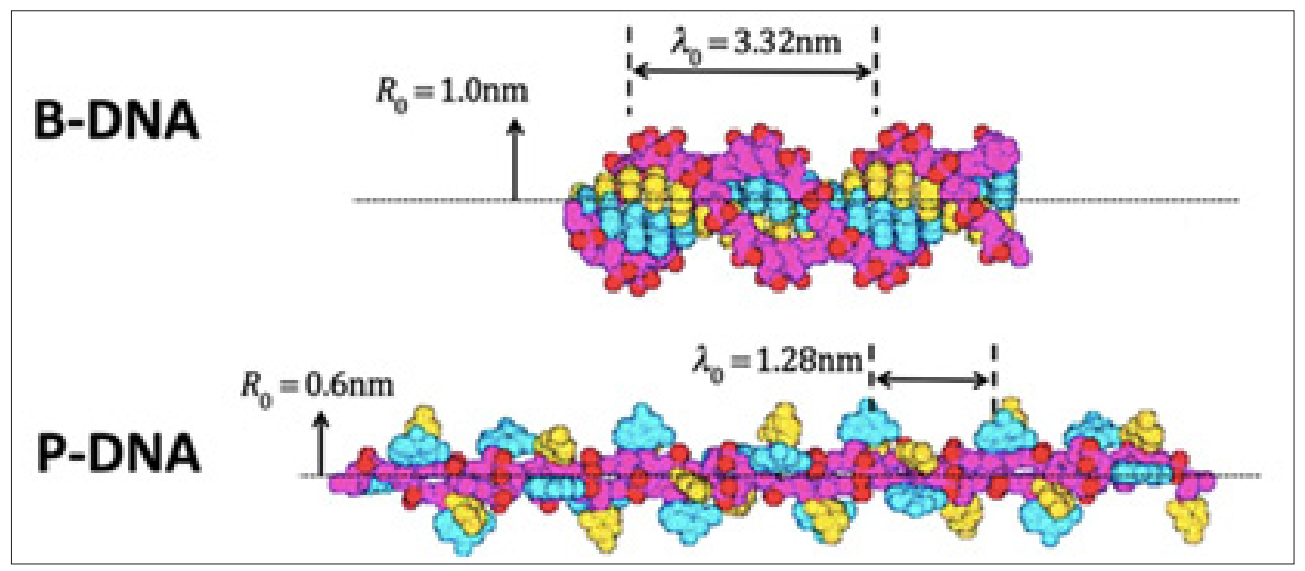

Figure 12.

If we want to see if our theory could predict things in the real world, and decided to try and calculate how much energy you'd need to convert one form of DNA to another. DNA is after all a spiral and a form of information. We needed to know the entropy of the galaxy for our calculations. Luckily, the mathematical physicist Roger Penrose showed that this entropy is dominated by the entropy of its central super-massive black hole. We know the mass of this black hole ( $4.3 \mathrm{~m}$ sun masses). And amazingly, when you know the mass of a black hole, there is an equation, discovered by the late physicist Stephen Hawking, that calculates its entropy. Hawking also discovered how to calculate the "temperature" at its surface, or "event horizon".

If you can assign a "temperature" to the black hole event horizon-which has no stuff in it to have temperature-why not also assign a temperature to a galaxy? We argue in our paper that this is reasonable (using what's known as the "holographic principle"). So we used our info-entropy equations to calculate the galaxy's holographic temperature.

Then it gets easy. We know that the galactic energy is given by the product of its entropy and temperature. And when we know the energy we can find out the mass thanks to Einstein's famous equation: $\mathrm{E}=\mathrm{mc} 2$.

This time the result was not exactly spot on, but it was reasonably close given our highly simplified model of the galaxy. The info-entropic geometry of a galaxy not only explains how entropic forces create the beautifully symmetric shape and keep it, but also accounts for all the mass that appears to be evident in it.

We think that the "morphogenic field" Kepler was seeking really does exist, and is actually the effect of the intertwining of information and entropy. After four long centuries, it seems Kepler has finally been vindicated.

\section{References}

1. Holzhey C, Larsen F, F Wilczek (1994) Geometric and renormalized entropy in conformal field theory. Nucl Phys B 424(3): 443-467.

2. E Verlinde (2011) On the origin of gravity and the laws of Newton. J High Energy Phys (4): 29.
3. K Skenderis, M Taylor (467) The fuzzball proposal for black holes. Phys Rep 467(4-5): 117-171.

4. Blanco DD, Casini H, Hung LY, Myers RC (2013) Relative entropy and holography. J High Energy Phys (8): 60.

5. Raamsdonk M (2010) Building up spacetime with quantum entanglement. Gen Rel Gravit 42(10): 2323-2329.

6. Lashkari N, Dermott M, Raamsdonk M (2014) Gravitational dynamics from entanglement thermodynamics. J High Energy Phys (4): 195.

7. Resconi G, Licata I, Fiscaletti D (2013) Unification of quantum and gravity by nonclassical information entropy space. Entropy 15(9): 36023619.

8. Licata I, Chiatti L (2009) The archaic universe: big bang, cosmological term and the quantum origin of time in projective cosmology. Int J Theor Phys 48(4): 1003-1018.

9. Lamb WE, Ratherford RC (1947) Fine structure of the hydrogen atom by a microwave method. Phys Rev 72(3): 241-243.

10. Bethe HA (1947) The electromagnetic shift of energy levels. Phys Rev 72(4): 339-341.

11. Lamoreaux SK (1997) Demonstration of the casimir force in the 0.6 to 6mu m range. Phys Rev Lett 78(1): 5-8.

12. Stephens CR, Hooft GT, Whiting BF (1994) Black-hole evaporation without information loss. Class Quantum Grav 11(3): 621-647.

13. Susskind L (1995) The world as a hologram. J Math Phys 36(11): 6377. 6396.

14. R Bousso (2002) The holographic principle. Rev Mod Phys 74(3): 825874.

15. Hawking SW (1975) Particle creation by black holes. Commun Math Phys 43(3): 199-220

16. Shujuan Liu, Hongwei X (2019) On the quantum thermodynamic origin of gravitational force by applying spacetime entanglement entropy and Unruh effect.

17. Skopec R (2019) Quantum resurrection: Quantum algorithm with complex conjugation reverses phases of the wave function components. Journal of Neuroscience and Neurological Surgery.

18. Skopec R (2018) Evolution continues with quantum biology and artificial intelligence. ARC Journal of Immunology and Vaccines 3(2): 15-23.

19. Skopec R (2019) Naphazoline nitrate treat the frey effect of microwave and other sonic weapon's damages in human's internal, organs. Virology: Research \& Reviews 2(1): 1-5. 
20. Skopec R (2019) Negative health effects of the international space station. Stem Cell Research International 3(2): 1-6.

21. Skopec R (2019) Fifth dark force completely change our understanding of the universe. Research Journal of Nanoscience and Engineering 3(2) 22-29.

22. Skopec R (2019) Darwin's theorem revised: Survival of the careerist. Advancements in Cardiovascular Research 1(5): 89-93.
23. Skopec R (2019) New psychological weapons make targets hallucinate. Journal of Neuropsychiatry and Neuro disorders 1(1): 1-6.

24.Skopec R (2019) The transfiguration with self-phase modulation effect of entanglement in a plasmatic moving frame. Current Trends in Biotechnology and Biochemistry.

For possible submissions Click below: 